\title{
Zero Vector and AT Math
}

\section{Paul T E Cusack*}

BScE, DULE, 23 Park Ave, Saint John, NB E2J 1R2, Canada

\section{Article Info}

\section{*Corresponding author:}

Paul T E Cusack

BScE, DULE

23 Park Ave

Saint John, NB E2J 1R2

Canada

Tel: +1-506-652-6350

E-mail: St-michael@hotmail.com

Received: November 28, 2018

Accepted: December 20, 2018

Published: January 17, 2019

Citation: Cusack PTE. Zero Vector and AT Math. Int J Cosmol Astron Astrophys. 2019; 1(1): 16-17.

doi: 10.18689/ijcaa-1000106

Copyright: (c) 2019 The Author(s). This work is licensed under a Creative Commons Attribution 4.0 International License, which permits unrestricted use, distribution, and reproduction in any medium, provided the original work is properly cited.

Published by Madridge Publishers

\begin{abstract}
From Linear Algebra we have a vector called the aero vector. It has interesting properties that lead to fundamental universal constants: the golden mean parabola; the gravitational constant, the super force and moment.
\end{abstract}

Keywords: Zero Vector; Astrotheology; Linear Algebra

\section{Introduction}

The Zero Vector $(0,0,0 \ldots . .0)$ is an interesting vector. It is perpendicular to every other vector and to itself. From this, we can derive the golden mean parabola; the gravitational constant, the super force and moment. We begin with the aero vector [1-3].

Given that:

$\{0\} \neg \lambda\left\{a_{1}, a_{2^{\prime}}, \ldots . a_{\infty}\right\}$

$\{0\} \neg\{0\}$

Then:

$\{0\}=\sum \lambda\left\{a_{1}, a_{2}, \ldots . a_{\infty}\right\}$

$=\lambda \int\left\{a_{1}, a_{2}, \ldots . a_{\infty}\right\}$

$=\lambda \infty$

$\sum \lambda\left\{a_{1}, a_{2}, \ldots . a_{\infty}\right\}=\cos (\pi / 2)=0$

$[\cos \theta]^{\prime}=\sin \theta$

$\sin (\pi / 2)=1$

$\cos (\pi / 2) \neg \int \sin (\pi / 2)=\int 1+\mathbb{C} 1$

Now,

$$
\begin{aligned}
& \sum \lambda\left\{a_{1}, a_{2^{\prime}} \ldots a_{\infty}\right\}=0 \\
& \rightarrow \lambda=0 \text { or }\left\{a_{1}, a_{2^{\prime}} \ldots . a_{\infty}\right\}=0 \\
& \sum \lambda\left\{a_{1}, a_{2^{\prime}} \ldots . a_{\infty}\right\}=\int 1+\mathbb{C} 1 \\
& \lambda \neq 0 \text { or } \lambda\left\{a_{1}, a_{2^{\prime}} \ldots . a_{\infty}\right\}=\int 1 \\
& \text { Let } y=y^{\prime} \\
& \int A=1 \\
& a^{2} / 2=1 \\
& A=\sqrt{ } 2
\end{aligned}
$$

And,

$\int A=\int 1$

$1 / 2 A^{2}=A+\mathbb{C} 2$

$A^{2}-A-1=0$ 
Golden Mean Parabola

$$
\begin{aligned}
& A^{2} / 2=A+\mathbb{C} 1 \\
& A^{2}=2 A \\
& A=2 \\
& A=\{2,0,0, \ldots .0\} \\
& L=\sqrt{ }\left[a_{1}{ }^{2}+a_{2}{ }^{2}+. . a_{\infty}{ }^{2}\right] \\
& 2^{2}=\left[a_{1}{ }^{2}+a_{2}{ }^{2}+. . a_{\infty}{ }^{2}\right] \\
& a_{1}=2 \\
& \text { Circ. }=\text { Area' } \\
& 2 \pi R=\pi R^{2} \\
& R=2 \\
& =a \\
& =d M / d t
\end{aligned}
$$

Pythagoras \& Equation of a Circle

$$
\begin{aligned}
& a^{2}+b^{2}=R^{2} \\
& \sqrt{ } 2^{2}+\sqrt{ } 2^{2}=2^{2}
\end{aligned}
$$

Consider:

$$
\begin{aligned}
& \int\left(a^{2}+b^{2}\right)=R^{2} \\
& a^{3} / 3+b^{3} / 3=R^{3} / 3 \\
& a^{3} / 3+b^{3} / 3+2^{3} / 3 \\
& a=b \\
& 2 a^{3} / 3=8 / 3 \\
& G(8)=\text { S.F. } \\
& 2 a^{3}=8 \\
& a=\sqrt[3]{4}=1.587 \\
& =1 \text {-sin } 1 \\
& =\text { Moment }
\end{aligned}
$$

Because the Zero Vector Space is finite, the universe is finite.

\section{Conclusion}

In the aero vector we see a convergence on a solution that gives us the universal constants and equations. It is the oddities that belie the truth about math and physics.

\section{References}

1. Cusack P. Astro-Theology, Cusacks Universe. J. Phys. Math. 2016; 7(2): 8.

2. Steward I. In Pursuit of the Unknown. NY 2012.

3. Axler S. Linear Algebra Done Right. Springer. 2015. 